\title{
Prevention of Leptospirosis Infected Vector and Human Population by Multiple Control Variables
}

\author{
Muhammad Altaf Khan, ${ }^{1}$ Saeed Islam, ${ }^{1}$ Sher Afzal Khan, ${ }^{2}$ \\ Ilyas Khan, ${ }^{3,4}$ Sharidan Shafie, ${ }^{3}$ and Taza Gul ${ }^{1}$ \\ ${ }^{1}$ Department of Mathematics, Abdul Wali Khan University, Mardan, Khyber Pakhtunkhwa, Pakistan \\ ${ }^{2}$ Department of Computer Sciences, Abdul Wali Khan University, Mardan, Khyber Pakhtunkhwa, Pakistan \\ ${ }^{3}$ Department of Mathematical Sciences, Faculty of Science, Universiti Teknologi Malaysia, Skudai, Johor, Malaysia \\ ${ }^{4}$ College of Engineering, Majmaah University, Majmaah, Saudi Arabia
}

Correspondence should be addressed to Sharidan Shafie; ridafie@yahoo.com

Received 28 April 2014; Accepted 25 June 2014; Published 25 November 2014

Academic Editor: Yasir Khan

Copyright (c) 2014 Muhammad Altaf Khan et al. This is an open access article distributed under the Creative Commons Attribution License, which permits unrestricted use, distribution, and reproduction in any medium, provided the original work is properly cited.

\begin{abstract}
Leptospirosis is an infectious disease that damages the liver and kidneys, found mainly in dogs and farm animals and caused by bacteria. In this paper, we present the optimal control problem applied to a dynamical leptospirosis infected vector and human population by using multiple control variables. First, we show the existence of the control problem and then use analytical and numerical techniques to investigate the existence cost effective control efforts for prevention of indirect and direct transmission of this disease. In order to do this, we consider three control functions two for human and one for vector population. We completely characterize the optimal control problem and compute the numerical solution of the optimality system using an iterative method.
\end{abstract}

\section{Introduction}

Leptospirosis disease is a globally important infectious disease. The disease is caused by a bacteria which is called Leptospira. Human as well as cattle is mostly infected from this disease [1]. The human is infected by means of drinking the water in which a rat was found dead, and cattle that drink this water become infectious. The human whose urine is used by other animals and cattle is also infected because the leptospirosis disease germs come out in urine. Those who wade through dirty water are mostly infected from this disease. Weil's first time described leptospirosis as a unique disease process in 1886, while 30 years before Inada and his colleagues identified the causal organism. The symptoms of leptospirosis are high fever, headache, chills, muscle aches, conjunctivitis (red eyes), diarrhea, vomiting, and kidney or liver problems (which may also include jaundice), anemia, and, sometimes, rash. Symptoms may last from a few days and up to several weeks. Deaths from this disease may occur, but they are rare. For some cases the infections can be mild and without obvious symptom [2-6].
Many models have been proposed to represent the dynamics of both human and vector population [7-9]. Pongsuumpun et al. [10] developed mathematical models to study the behavior of leptospirosis disease. They represent the rate of change for both rats and human population. The human population is further divided into two main groups: juveniles and adults. Triampo et al. [11] considered a deterministic model for the transmission of leptospirosis disease [11]. In their work they considered a number of leptospirosis infections in Thailand and showed the numerical simulations. Zaman [12] considered the real data presented in [11] to study the dynamical behavior and role of optimal control theory. The dynamical interaction including local and global stability of leptospirosis infected vector and human population can be found in Zaman et al. [13]. In their work they also presented the bifurcation analysis and presented the numerical simulations for different values of infection rate. In [14], the author presented an epidemic model of malaria, by using three control variables, and obtained their optimal solutions; for more references, see, for example, [14-17]. 
In this paper, we consider the basic model studied in [13] to incorporate some important epidemiological features. We use optimal control theory to reduce the proportion of the infected human and infected vector population by using multiple control. At the long-term level of infected human, every infected human on average causes one further secondary case. Therefore, if we can reduce the number of infected humans further, the disease does less well and increase the recovered human. Here we define the control variables; the first control is to cover all cuts and wear dry, full-cover boots, shoes and long sleeve shirts when handling animals. The second control represents washing hands thoroughly on a regular basis and showering after work. Cleaning up both work place and home is our third control. To do this, we first show the existence of the optimal control system. Then, by solving the optimality system analytically, which consists of the original state system, the adjoint system and their boundary conditions. The real data presented for leptospirosis epidemic in Thailand have been used in numerical simulations. We also conclude by discussing the results of the numerical simulations for our epidemic mathematical model.

The structure of the paper is organized as follows. Section 2 is devoted to the mathematical formulation and solution of the problem which is further divided into the following subsections. Section 2.1 includes the formulation of the mathematical model followed by Section 2.2 which contains optimal control problem whereas Section 2.3 explains the bifurcation analysis of the control problem. The last Section 2.4 discusses the existence of control problem. Section 3 is devoted to the numerical solution of the optimality system. Finally, the conclusion is presented in Section 4.

\section{Mathematical Formulation and Solution}

2.1. Mathematical Model. In this section, a vector-host epidemic model with direct transmission is presented. The host population at time $t$ is divided into susceptible $S_{h}(t)$, $I_{h}(t)$ infected and recovered $R_{h}(t)$ individuals. The vector (rats) populations at time $t$ is divided into susceptible $S_{v}(t)$ and infected vector population $I_{v}(t)$. The total population of human is denoted by $N_{h}$ and the total population of the vector is denoted by $N_{v}$. Thus, $N_{h}(t)=S_{h}(t)+I_{h}(t)+R_{h}(t)$ and $N_{v}(t)=S_{v}(t)+I_{v}(t)$. The mathematical representation of the model which consists of the system of nonlinear differential equations with five state variables is given by

$$
\begin{aligned}
& \frac{d S_{h}}{d t}=b_{1}-\mu_{h} S_{h}-\beta_{2} S_{h} I_{v}-\beta_{1} S_{h} I_{h}+\lambda_{h} R_{h}, \\
& \frac{d I_{h}}{d t}=\beta_{2} S_{h} I_{v}+\beta_{1} S_{h} I_{h}-\mu_{h} I_{h}-\delta_{h} I_{h}-\gamma_{h} I_{h}, \\
& \frac{d R_{h}}{d t}=\gamma_{h} I_{h}-\mu_{h} R_{h}-\lambda_{h} R_{h},
\end{aligned}
$$

$$
\begin{aligned}
& \frac{d S_{v}}{d t}=b_{2}-\gamma_{v} S_{v}-\beta_{3} S_{v} I_{h}, \\
& \frac{d I_{v}}{d t}=\beta_{3} S_{v} I_{h}-\gamma_{v} I_{v}-\delta_{v} I_{v} .
\end{aligned}
$$

Here $b_{1}$ is the recruitment rate of human population, susceptible human can be infected by two ways of transmission, that is, directly or through infected individuals, and $\beta_{1}, \beta_{2}$ are the mediate transmission coefficients. $\mu_{h}$ is the natural mortality rate for human; $\gamma_{h}$ is the recovery rate for human from the infections. We assumed that disease may be fatal to some infectious host, so disease related death rate from infected class occurs at human populations at $\delta_{h}$. The immune human once again is susceptible at constant rate $\lambda_{h} . b_{2}$ is the recruitment rate for vector population. The infectious vector dies due to disease at vector populations at the rate of $\delta_{v} \cdot \beta_{3}$ represents the disease carrying of susceptible vector per host per unit time; $\gamma_{v}$ is the death rate of vector.

2.2. Optimal Control Problem. Optimal control theory is a powerful mathematical tool which makes the decision involving complex dynamical systems [18]. Optimal control method has been used to study the dynamics of the disease; we refer the reader to [19-21]; no such method is used, according to the author's knowledge, to determine optimal control measure for vector-host epidemic direct transmission. The problem is to minimize the infected human and vector population and to maximize the recovered human population. In the system (1) we have five state variables $S_{h}(t)$, $I_{h}(t), R_{h}(t), S_{v}(t)$, and $I_{v}(t)$. In this optimal control problem we use three control variables.

(i) The first control $u_{1}(t)$ (Figure 7) represents that human should cover all cuts, abrasions with waterproof dressing, grazes, wear dry clothes, wear fullcover shoes, gloves, and use the shirts with long sleeves when handling the animals.

(ii) The second control $u_{2}(t)$ (Figure 8) shows that after work human should bath or shower regularly and adopt the habit of washing hands regularly.

(iii) Our third control $u_{3}(t)$ (Figure 9) represents cleaning the home and working area.

In the human population, the associated force of infections are reduced by factors of $\left(1-u_{1}(t)\right)$ and $\left(1-u_{2}(t)\right)$, respectively. We assume that the mortality rate of vector population increases at a rate proportional to $u_{3}(t)$, where $\epsilon_{1}>0$ and $\epsilon_{2}>0$ are rate constants. Taking into account the extensions and assumptions made above, it follows that the dynamics of the system (1) are governed by the following system of five differential equations:

$$
\begin{aligned}
\frac{d S_{h}}{d t}= & b_{1}-\mu_{h} S_{h}-\beta_{2} S_{h} I_{v}\left(1-u_{1}(t)\right) \\
& -\beta_{1} S_{h} I_{h}\left(1-u_{2}(t)\right)+\lambda_{h} R_{h}
\end{aligned}
$$




$$
\begin{aligned}
\frac{d I_{h}}{d t}= & \beta_{2} S_{h} I_{v}\left(1-u_{1}(t)\right)+\beta_{1} S_{h} I_{h}\left(1-u_{2}(t)\right) \\
& -\mu_{h} I_{h}-\delta_{h} I_{h}-\gamma_{h} I_{h}-u_{2}(t) I_{h}, \\
\frac{d R_{h}}{d t}= & \gamma_{h} I_{h}-\mu_{h} R_{h}-\lambda_{h} R_{h}+u_{1}(t) S_{h}+u_{2}(t) I_{h}, \\
\frac{d S_{v}}{d t}= & b_{2}-\gamma_{v} S_{v}-\beta_{3} S_{v} I_{h}-\epsilon_{1} u_{3}(t) S_{v} \\
\frac{d I_{v}}{d t}= & \beta_{3} S_{v} I_{h}-\gamma_{v} I_{v}-\delta_{v} I_{v}-\epsilon_{2} u_{3}(t) I_{v}
\end{aligned}
$$

with initials conditions

$$
\begin{array}{ll}
S_{h}(0) \geq 0, & I_{h}(0) \geq 0, \quad R_{h}(0) \geq 0, \\
S_{v}(0) \geq 0, & I_{v}(0) \geq 0,
\end{array}
$$

where $A_{1}, A_{2}, B_{1}$, and $B_{2}$ are positive constants to keep the balance of the size of the individuals $S_{h}(t), I_{h}(t), S_{v}(t)$, and $I_{v}(t)$, respectively. For the optimal control problem we consider the control variable $u_{1} \in U$. Here, $u_{1}, u_{2}, u_{3} \in U$ is measurable; $0 \leq u_{1}(t), u_{2}(t), u_{3}(t) \leq 1$.

2.3. Bifurcation Analysis of the Control Problem. In this subsection, we present the endemic equilibria which is further used in the bifurcation analysis. In order to do this we set the left-hand side of the system (2) equal to zero and use the technique developed in [22], getting

$$
\begin{aligned}
S_{h}^{*}= & \frac{P_{1} P_{2}\left(\gamma_{v}+\beta_{3} I_{h}^{*}+\epsilon_{1} u_{3}\right)}{\beta_{2} \beta_{3} b_{2}\left(1-u_{1}\right)+P_{1} \beta_{1}\left(1-u_{2}\right)\left(\gamma_{v}+\beta_{3} I_{h}^{*}+\epsilon_{1} u_{3}\right)}, \\
S_{v}^{*}= & \frac{b_{2}}{\gamma_{v}+\beta_{3} I_{h}^{*}+\epsilon_{1} u_{3}}, \\
I_{v}^{*}= & \frac{\beta_{3} b_{2} I_{h}^{*}}{P_{1}\left(\gamma_{v}+\beta_{3} I_{h}^{*}+\epsilon_{1} u_{3}\right)}, \\
R_{h}^{*}= & \left(P _ { 3 } I _ { h } ^ { * } \left(\beta_{2} \beta_{3} b_{2}\left(1-u_{1}\right)\right.\right. \\
& \left.+P_{1} \beta_{1}\left(1-u_{2}\right)\left(\gamma_{v}+\beta_{3} I_{h}^{*}+\epsilon_{1} u_{3}\right)+u_{1}\right) \\
& \left.\times P_{1} P_{2}\left(\gamma_{v}+\beta_{3} I_{h}^{*}+\epsilon_{1} u_{3}\right)\right) \\
& \times\left(P _ { 4 } \left(\beta_{2} \beta_{3} b_{2}\left(1-u_{1}\right)+P_{1} \beta_{1}\left(1-u_{2}\right)\right.\right. \\
& \left.\left.\quad \times\left(\gamma_{v}+\beta_{3} I_{h}^{*}+\epsilon_{1} u_{3}\right)\right)\right)^{-1},
\end{aligned}
$$

where

$$
\begin{aligned}
& P_{1}=\gamma_{v}+\delta_{v}+\epsilon_{2} u_{3}, \\
& P_{2}=\mu_{h}+\delta_{h}+\gamma_{h}+u_{2}, \\
& P_{3}=\mu_{h}+\lambda_{h} .
\end{aligned}
$$

The reproduction number for the control system (2) is given by

$$
K_{u}=\frac{b_{1}}{\mu_{h}}\left(\frac{\beta_{2} \beta_{3} b_{2}\left(1-u_{1}\right)}{P_{1} P_{2}\left(\gamma_{v}-\epsilon_{1} u_{3}\right)}+\frac{\beta_{1}\left(1-u_{2}\right)}{P_{2}}\right) .
$$

The reproduction number for without control system is given by

$$
K_{0}=\frac{b_{1}}{\mu_{h}}\left(\frac{\beta_{2} \beta_{3} b_{2}}{\gamma_{v} P_{0}^{1} P_{0}^{2}}+\frac{\beta_{1}}{P_{0}^{2}}\right)
$$

where $P_{0}^{1}=\left(\delta_{v}+\gamma_{v}\right)$ and $P_{0}^{2}=\left(\mu_{h}+\delta_{h}+\gamma_{h}\right)$. In the above expression for the endemic equilibria the infected component $I_{h}^{*}$ is nonzero. Using the value of $S_{h}^{*}, R_{h}^{*}$, and $I_{v}^{*}$ in the first equation of the system (2), we obtained

$$
f\left(I_{h}^{*}\right)=a I_{h}^{* 2}+b I_{h}^{*}+c=0
$$

where

$$
\begin{aligned}
a= & \lambda_{h} \beta_{3}^{2} P_{1} \beta_{1}\left(1-u_{2}\right) \\
b= & \lambda_{h} \beta_{3}^{2} \beta_{2} b_{2}\left(1-u_{1}\right)+P_{4} b_{1} \beta_{1} P_{1}\left(1-u_{2}\right) \beta_{3} \\
& +\lambda_{h} \beta_{3} P_{1}\left(\gamma_{v}+\epsilon_{1} u_{3}\right) \beta_{1}\left(1-u_{2}\right) \\
& -P_{2} P_{4} \beta_{2}\left(1-u_{1}\right) \beta_{3} \beta_{2} b_{2}-\beta_{1}\left(1-u_{2}\right) P_{1} \beta_{3} \\
& +u_{1} \lambda_{h} P_{1} \beta_{3}, \\
c= & -P_{1} P_{2} P_{4}\left(\gamma_{v}+\epsilon_{1} u_{3}\right) \mu_{h}\left(\beta_{1}\left(1-u_{2}\right)-u_{1} \lambda_{h}\right)\left(1-K_{u}\right) .
\end{aligned}
$$

Here the coefficient $a$ is positive always and $c$ depends upon the value of $K_{u}$; if the value of $K_{u}<1$, then $c$ is positive; otherwise negative. The positive solution of the above equation depends upon the value of $b$ and $c$. For the value of $K_{u}>1$, the above equation leads to two roots, positive and negative. If we substitute $K_{u}=1$, then the equation has no positive solution. This is possible if and only if $b<0$. For $b<0$ and $K_{u}=1$, the equilibria depend upon $K_{u}$; then there exists an open interval having two positive roots, that is, $I_{1}=\left(-b-\sqrt{b^{2}-4 a c}\right) / 2 a$ and $I_{2}=(-b+$ $\left.\sqrt{b^{2}-4 a c}\right) / 2 a$. For $c>0$ either $b^{2}<4 a c$ or $b \geq 0$, then the above have no positive solution.

For backward bifurcation, we set $b^{2}-4 a c=0$ and $K_{u}=R_{c}$ and solve for the critical value of $K_{c}$, which is given by

$$
R_{c}=1-\frac{b^{2}}{4 a P_{1} P_{2} P_{4}\left(\gamma_{v}+\epsilon_{1} u_{3}\right) \mu_{h}\left(\beta_{1}\left(1-u_{2}\right)-u_{1} \lambda_{h}\right)} .
$$

It is proved by simulating the set of parameter values presented in Table 1. Figure 1 shows the bifurcation of the control system (2). The high bold black line shows the bifurcation at $u_{1}=u_{2}=u_{3}=0$, that is, without control. The small black bold line shows the bifurcation at $u_{1}=u_{2}=u_{3} \neq 0$, that is, with control. The change occurs in the bifurcation rapidly 
TABLE 1: Parameter values used in the control bifurcation.

\begin{tabular}{lc}
\hline Notation & Value \\
\hline$b_{2}$ & 10 \\
$\beta_{1}$ & 0.048 \\
$\beta_{3}$ & 0.006 \\
$u_{2}$ & 0.001 \\
$\lambda_{h}$ & 0.068 \\
$\gamma_{h}$ & 0.041 \\
$b_{1}$ & 10 \\
$\beta_{2}$ & 0.0008 \\
$u_{1}$ & 0.541 \\
$u_{3}$ & 0.081 \\
$\mu_{h}$ & 0.091 \\
$\delta_{h}$ & 0.10 \\
$\delta_{v}$ & 0.009 \\
$\epsilon_{2}$ & 0.009 \\
$\epsilon_{1}$ & 0.00001 \\
$\gamma_{v}$ & 0.087 \\
\hline
\end{tabular}

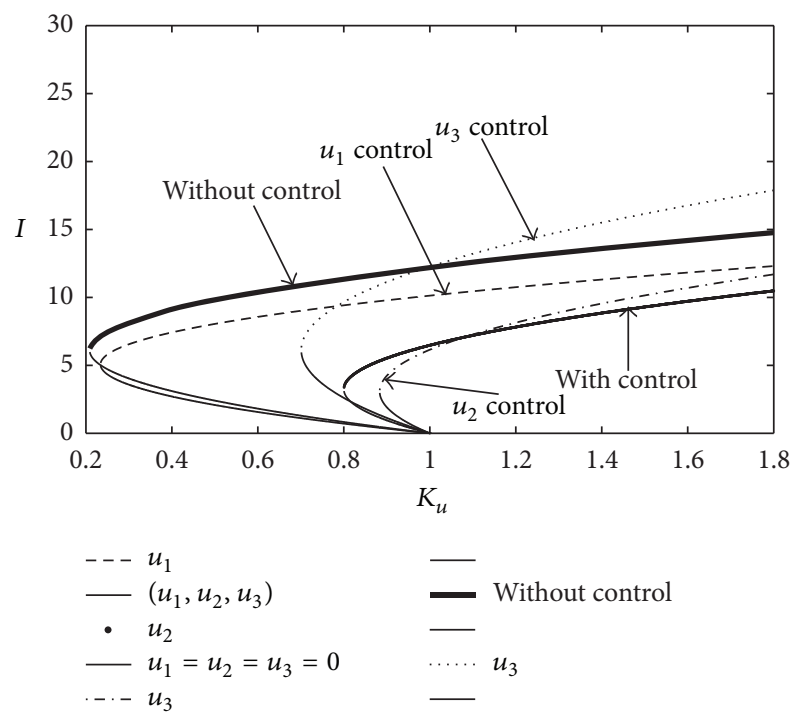

FIGURE 1: The plot shows the backward bifurcation for control and without control system. when we apply the control variable. $u_{1}$ is the first control, which is represented by the bold dotted line, the dotted dashed line represents the second control, which shows the change in the control, the very narrow dotted line represent the third control, and bifurcation occurs for the control variable.

2.4. Existence of Control Problem. We use the bounded Lebesgue measurable control and define our objective functional as

$$
\begin{gathered}
J\left(u_{1}, u_{2}, u_{3}\right) \\
=\min \int_{0}^{T}\left(A_{1} S_{h}(t)+A_{2} I_{h}(t)+B_{1} S_{v}(t)+B_{2} I_{v}(t)\right. \\
\left.\quad+\frac{1}{2}\left(W_{1} u_{1}^{2}+W_{2} u_{2}^{2}+W_{3} u_{3}^{2}\right)\right) d t .
\end{gathered}
$$

Here, in (11), $A_{1}, A_{2}, B_{1}, B_{2}, W_{1}, W_{2}$, and $W_{3}$ represent the weight/balance factors just to keep the balance of individuals in the objective functional. The control set is defined as

$$
\begin{gathered}
U=\left\{u=\left(u_{1}, u_{2}, u_{3}\right) \mid u_{i}(t)\right. \text { is Lebesgue measureable, } \\
\left.0 \leq u_{i}(t) \leq 1, t \in[0, T], \text { for } i=1,2,3\right\} .
\end{gathered}
$$

Note. The details of the control variables $u_{1}, u_{2}$, and $u_{3}$ are available in Section 2.2.

First, we show the existence for the control system (2). Let $S_{h}(t), I_{h}(t), R_{h}(t), S_{v}(t)$, and $I_{v}(t)$ be the state variables with control variables $u_{1}(t), u_{2}(t)$, and $u_{3}(t)$. For existence we consider the control system (2). Then we can write the system (2) in the following form:

$$
X^{\prime}=A X+F(X)
$$

where

$$
\begin{gathered}
X^{\prime}=\left[\begin{array}{c}
S_{h}(t) \\
I_{h}(t) \\
R_{h}(t) \\
S_{v}(t) \\
I_{v}(t)
\end{array}\right], \\
A=\left[\begin{array}{cccc}
-\mu_{h} & \lambda_{h} & 0 & 0 \\
0 & -\mu_{h}-\delta_{h}-\gamma_{h}-u_{2}(t) & 0 & 0 \\
\gamma_{h}+u_{2}(t) & -\mu_{h}-\lambda_{h} & 0 & 0 \\
0 & 0 & -\gamma_{v}-\epsilon_{1} u_{3}(t) & 0 \\
0 & 0 & 0 & -\gamma_{v}-\delta_{v}-\epsilon_{2} u_{3}(t)
\end{array}\right], \\
F(X)=\left[\begin{array}{cc}
0 \\
b_{1}-\beta_{2} S_{h}(t) I_{v}\left(1-u_{1}(t)\right)-\beta_{1} S_{h}(t) I_{h}(t)\left(1-u_{2}(t)\right) \\
\beta_{2} S_{h}(t) I_{v}\left(1-u_{1}(t)\right)+\beta_{1} S_{h}(t) I_{h}(t)\left(1-u_{2}(t)\right)\left(1-u_{2}(t)\right) \\
0 \\
0
\end{array}\right], \beta_{3} S_{v}(t) I_{h}(t)
\end{gathered}
$$


where $X^{\prime}$ denotes the derivative with respect to time $t$. The system (13) is a nonlinear system with a bounded coefficient. We set

$$
G(X)=A X+F(X) .
$$

The second term on the right-hand side of (15) satisfies

$$
\begin{aligned}
& \left|F\left(X_{1}\right)-F\left(X_{2}\right)\right| \\
& \leq C_{1}\left(\left|\left(S_{1 h}(t)-S_{2 h}(t)\right)\right|+C_{2}\left|\left(I_{1 h}(t)-I_{2 h}(t)\right)\right|\right. \\
& \quad+C_{3}\left|\left(R_{1 h}(t)-R_{2 h}(t)\right)\right|+C_{4}\left|\left(S_{1 v}(t)-S_{2 v}(t)\right)\right| \\
& \left.\quad+C_{5}\left|\left(I_{1 v}(t)-I_{2 v}(t)\right)\right|\right) \\
& \leq C\left(\left|\left(S_{1 h}(t)-S_{2 h}(t)\right)\right|+\left|\left(I_{1 h}(t)-I_{2 h}(t)\right)\right|\right. \\
& +\left|\left(R_{1 h}(t)-R_{2 h}(t)\right)\right|+\left|\left(S_{1 v}(t)-S_{2 v}(t)\right)\right| \\
& \left.+\left|\left(I_{1 v}(t)-I_{2 v}(t)\right)\right|\right)
\end{aligned}
$$

where the positive constant $C=\max \left(C_{1}, C_{2}, C_{3}, C_{4}, C_{5}\right)$ is independent of the state variables. Also we have

$$
\left|G\left(X_{1}\right)-G\left(X_{2}\right)\right| \leq C\left|X_{1}-X_{2}\right|,
$$

where $C=C_{1}+C_{2}+C_{3}+C_{4}+C_{5}+\|M\|<\infty$. So, it follows that the function $G$ is uniformly Lipschitz continuous. From the definition of control variables and nonnegative initial conditions we can see that a solution of the system (13) exists; see [23]. Now, we consider the control system (2) with the initial conditions (3) to show the existence of the control problem. Note that for bounded Lebesgue measurable controls and nonnegative initial conditions, nonnegative bounded solutions to the state system exist [23]. Let us go back to the optimal control problem (2)-(3). In order to find an optimal solution, first we will find the Lagrangian $L$ and Hamiltonian $H$ for the optical control problem (2)-(3). The Lagrangian for our control problem is given by

$$
\begin{aligned}
L= & A_{1} S_{h}+A_{2} I_{h}+B_{1} S_{v}+B_{2} I_{v} \\
& +\frac{1}{2}\left(W_{1} u_{1}^{2}+W_{2} u_{2}^{2}+W_{3} u_{3}^{2}\right) .
\end{aligned}
$$

For the minimum value of Lagrangian, we define the Hamiltonian for the control problem:

$$
\begin{aligned}
H= & L\left(S_{h}, I_{h}, S_{v}, I_{v}, u_{1}, u_{2}, u_{3}\right) \\
& +\lambda_{1} \frac{d S_{h}}{d t}+\lambda_{2} \frac{d I_{h}}{d t}+\lambda_{3} \frac{d R_{h}}{d t}+\lambda_{4} \frac{d S_{v}}{d t}+\lambda_{5} \frac{d I_{v}}{d t} .
\end{aligned}
$$

For the existence of our control problem, we state and prove the following theorem.

Theorem 1. There exists an optimal control $u^{*}=\left(u_{1}^{*}, u_{2}^{*}, u_{3}^{*}\right) \in$ U such that

$$
J\left(u_{1}^{*}, u_{2}^{*}, u_{3}^{*}\right)=\min _{\left(u_{1}, u_{2}, u_{3}\right) \in U} J\left(u_{1}, u_{2}, u_{3}\right),
$$

subject to the control system (2) with the initial conditions (3).
Proof. To prove the existence of an optimal control, we use the result in [24]; the control and the state variable are nonnegative values. In this minimizing of the problem, the necessary convexity of the objective functional in $u_{1}, u_{2}$, and $u_{3}$ is satisfied. The set of control variables $\left(u_{1}, u_{2}, u_{3}\right) \in U$ is also convex and closed by the definition. The optimal system is bounded which determines the compactness needed for the existence of optimal control. The integrand in the objective functional (11) is $A_{1} S(t)+A_{2} I(t)+B_{1} S_{v}+B_{2} I_{v}+(1 / 2)\left(W_{1} u_{1}^{2}+\right.$ $\left.W_{2} u_{2}^{2}+W_{3} u_{3}^{2}\right)$ which is convex in the control set $U$. Also we can easily see that there exist a constant $\sigma>1$ and positive numbers $\omega_{1}$ and $\omega_{2}$ such that

$$
J\left(u_{1}, u_{2}, u_{3}\right) \geq \omega_{1}\left(\left|u_{1}\right|^{2}+\left|u_{2}\right|^{2}+\left|u_{3}\right|^{2}\right)^{\sigma / 2}-\omega_{2},
$$

which is the existence of an optimal control problem.

To find the optimal solution, we apply Pontryagin's maximum principle [25] given by the following.

If $(x, u)$ is an optimal solution for an optimal control problem, then there exists a nontrivial vector function $\lambda=$ $\left(\lambda_{1}, \lambda_{2}, \ldots, \lambda_{n}\right)$ which satisfies the following inequalities:

$$
\begin{aligned}
\frac{d x}{d t} & =\frac{\partial H(t, x, u, \lambda)}{\partial \lambda}, \\
0 & =\frac{\partial H(t, x, u, \lambda)}{\partial u}, \\
\frac{d \lambda}{d t} & =-\frac{\partial H(t, x, u, \lambda)}{\partial x} .
\end{aligned}
$$

Now we apply the necessary conditions to the Hamiltonian $H$ in (19).

Theorem 2. Suppose that $S_{h}^{*}, I_{h}^{*}, R_{h}^{*}, S_{v}^{*}$ and $I_{v}^{*}$ are the optimal state solutions with associated optimal control variables $\left(u_{1}^{*}, u_{2}^{*}, u_{3}^{*}\right)$ for the optimal control problem (2)-(3). Then there exist adjoint variables $\lambda_{i}$, for $i=1,2, \ldots, 5$, satisfying

$$
\begin{aligned}
\frac{d \lambda_{1}}{d t}= & \left(\lambda_{1}-\lambda_{2}\right)\left(\beta_{2} I_{v}\left(1-u_{1}\right)+\beta_{1} I_{h}\left(1-u_{2}\right)\right) \\
& +\lambda_{1} \mu_{h}-u_{1} \lambda_{3}-A_{1} \\
\frac{d \lambda_{2}}{d t}= & \left(\lambda_{1}-\lambda_{2}\right) \beta_{1} S_{h}\left(1-u_{2}\right)+\mu_{h} \lambda_{2}+\delta_{h} \lambda_{2}+\gamma_{h} \lambda_{2} \\
& +u_{2} \lambda_{2}-\lambda_{3} \gamma_{h}-\lambda_{3} u_{2}+\beta_{3} S_{v}\left(\lambda_{4}-\lambda_{5}\right)-A_{2}, \\
\frac{d \lambda_{3}}{d t}= & \left(\lambda_{3}-\lambda_{1}\right) \lambda_{h}+\mu_{h} \lambda_{3}, \\
\frac{d \lambda_{4}}{d t}= & \left(\lambda_{4}-\lambda_{5}\right) \beta_{3} I_{h}+\lambda_{4} \gamma_{v}+\lambda_{4} \epsilon_{1} u_{3}-B_{1} \\
\frac{d \lambda_{5}}{d t}= & \left(\lambda_{1}-\lambda_{2}\right) \beta_{2} S_{h}\left(1-u_{1}\right) \\
& -\lambda_{5} \mu_{v}+\lambda_{5} \gamma_{v}+\lambda_{5} \delta_{v}+\lambda_{5} \epsilon_{2} u_{3}-B_{2}
\end{aligned}
$$

with transversality conditions (or boundary conditions)

$$
\lambda_{i}(T)=0, \quad i=1,2, \ldots, 5 .
$$


Furthermore, optimal controls $u_{1}^{*}, u_{2}^{*}$, and $u_{3}^{*}$ are given by

$$
\begin{aligned}
& u_{1}^{*}=\max \left\{\min \left\{\frac{\left(\lambda_{2}-\lambda_{1}\right) \beta_{2} S_{h}^{*} I_{v}^{*}-\lambda_{3} S_{h}^{*}}{W_{1}}, 1\right\}, 0\right\}, \\
& u_{2}^{*}=\max \left\{\min \left\{\frac{\left(\lambda_{2}-\lambda_{1}\right) \beta_{1} S_{h}^{*} I_{h}^{*}+\left(\lambda_{2}-\lambda_{3}\right) I_{h}^{*}}{W_{2}}, 1\right\}, 0\right\}, \\
& u_{3}^{*}=\max \left\{\min \left\{\frac{\left(\lambda_{4} \epsilon_{1} S_{v}^{*}+\lambda_{5} \epsilon_{2} I_{v}^{*}\right)}{W_{3}}, 1\right\}, 0\right\} .
\end{aligned}
$$

Proof. To find the adjoint equations and the transversality conditions, we use the Hamiltonian (19). By setting $S_{h}(t)=$ $S_{h}^{*}(t), I_{h}(t)=I_{h}^{*}(t), R_{h}(t)=R_{h}^{*}(t), S_{v}(t)=S_{v}^{*}(t)$, and $I_{v}(t)=$ $I_{v}^{*}(t)$ and differentiating the Hamiltonian (19) with respect to $S_{h}, I_{h}, R_{h}, S_{v}$, and $I_{v}$, respectively, we get (23). Then solving the equations $\partial H / \partial u_{1}=0, \partial H / \partial u_{2}=0$, and $\partial H / \partial u_{3}=0$ on the interior of the control set and then using the optimality conditions and also the property of control space $U$, we can derive (25).

Here we call formulas (25) for the characterization of the optimal control. The optimal control and the state are determined by solving the optimality system, which consist of the state system (1), the adjoint system (23), initial conditions at (3), boundary conditions (24), and the characterization of the optimal control $\left(u_{1}^{*}, u_{2}^{*}, u_{3}^{*}\right)$ which is given by (25). In addition, the second derivative of the Lagrangian with respect to $u_{1}, u_{2}$, and $u_{3}$, respectively, is positive, which shows the minimum of the optimal control $u_{1}^{*}, u_{2}^{*}$, and $u_{3}^{*}$. Substituting the values of $u_{1}^{*}, u_{2}^{*}$, and $u_{3}^{*}$ in the control system (2), we obtain the following system:

$$
\begin{aligned}
& \frac{d S_{h}^{*}}{d t} \\
& =b_{1}-\mu_{h} S_{h}^{*}-\beta_{2} S_{h}^{*} I_{v}^{*} \\
& \quad \times\left(1-\max \left\{\min \left\{\frac{\left(\lambda_{2}-\lambda_{1}\right) \beta_{2} S_{h}^{*} I_{v}^{*}-\lambda_{3} S_{h}^{*}}{W_{1}}, 1\right\}, 0\right\}\right) \\
& -\beta_{1} S_{h}^{*} I_{h}^{*}\left(1-\max \left\{\min \left\{\frac{\left(\lambda_{2}-\lambda_{1}\right) \beta_{1} S_{h}^{*} I_{h}^{*}+\left(\lambda_{2}-\lambda_{3}\right) I_{h}^{*}}{W_{2}}, 1\right\}, 0\right\}\right) \\
& +\lambda_{h} R_{h}^{*}, \\
& \frac{d I_{h}^{*}}{d t} \\
& =\beta_{2} S_{h}^{*} I_{v}^{*}\left(1-\max \left\{\min \left\{\frac{\left(\lambda_{2}-\lambda_{1}\right) \beta_{2} S_{h}^{*} I_{v}^{*}-\lambda_{3} S_{h}^{*}}{W_{1}}, 1\right\}, 0\right\}\right) \\
& -\mu_{h} I_{h}^{*}-\delta_{h} I_{h}^{*}+\beta_{1} S_{h}^{*} I_{h}^{*} \\
& \quad \times\left(1-\max \left\{\min \left\{\frac{\left(\lambda_{2}-\lambda_{1}\right) \beta_{1} S_{h}^{*} I_{h}^{*}+\left(\lambda_{2}-\lambda_{3}\right) I_{h}^{*}}{W_{2}}, 1\right\}, 0\right\}\right) \\
& -\gamma_{h} I_{h}^{*}-\left(\max \left\{\min \left\{\frac{\left(\lambda_{2}-\lambda_{1}\right) \beta_{1} S_{h}^{*} I_{h}^{*}+\left(\lambda_{2}-\lambda_{3}\right) I_{h}^{*}}{W_{2}}, 1\right\}, 0\right\}\right) I_{h}^{*},
\end{aligned}
$$

$\frac{d R_{h}^{*}}{d t}$

$$
\begin{aligned}
= & \gamma_{h} I_{h}^{*}-\mu_{h} R_{h}^{*}-\lambda_{h} R_{h}^{*} \\
& +\left(\max \left\{\min \left\{\frac{\left(\lambda_{2}-\lambda_{1}\right) \beta_{2} S_{h}^{*} I_{v}^{*}-\lambda_{3} S_{h}^{*}}{W_{1}}, 1\right\}, 0\right\}\right) S_{h}^{*} \\
& +\left(\max \left\{\min \left\{\frac{\left(\lambda_{2}-\lambda_{1}\right) \beta_{1} S_{h}^{*} I_{h}^{*}+\left(\lambda_{2}-\lambda_{3}\right) I_{h}^{*}}{W_{2}}, 1\right\}, 0\right\}\right) I_{h}^{*},
\end{aligned}
$$

$\frac{d S_{v}^{*}}{d t}$

$$
\begin{aligned}
& =b_{2}-\gamma_{v} S_{v}^{*}-\beta_{3} S_{v}^{*} I_{h}^{*} \\
& -\epsilon_{1} S_{v}^{*}\left(\max \left\{\min \left\{\frac{\left(\lambda_{4} \epsilon_{1} S_{v}^{*}+\lambda_{5} \epsilon_{2} I_{v}^{*}\right)}{W_{3}}, 1\right\}, 0\right\}\right),
\end{aligned}
$$

$\frac{d I_{v}^{*}}{d t}$

$$
\begin{aligned}
= & \beta_{3} S_{v}^{*} I_{h}^{*}+\mu_{v} I_{v}^{*}-\gamma_{v} I_{v}^{*}-\delta_{v} I_{v}^{*} \\
& -\epsilon_{2} I_{v}^{*}\left(\max \left\{\min \left\{\frac{\left(\lambda_{4} \epsilon_{1} S_{v}^{*}+\lambda_{5} \epsilon_{2} I_{v}^{*}\right)}{W_{3}}, 1\right\}, 0\right\}\right)
\end{aligned}
$$

with Hamiltonian $H^{*}$ at $\left(t, S_{h}^{*}, I_{h}^{*}, R_{h}^{*}, S_{v}^{*}, I_{v}^{*}, u_{1}^{*}, u_{2}^{*}, u_{3}^{*}, \lambda_{1}\right.$, $\left.\lambda_{2}, \lambda_{3}, \lambda_{4}, \lambda_{5}\right)$ :

$$
\begin{aligned}
H^{*}=A_{1} & S_{h}^{*}+A_{2} I_{h}^{*}+B_{1} S_{v}^{*}+B_{2} I_{v}^{*} \\
+ & \frac{1}{2}\left(W_{1}\left(\max \left\{\min \left\{\frac{\left(\lambda_{2}-\lambda_{1}\right) \beta_{2} S_{h}^{*} I_{v}^{*}-\lambda_{3} S_{h}^{*}}{W_{1}}, 1\right\}, 0\right\}\right)^{2}+W_{2}\right. \\
& \times\left(\max \left\{\min \left\{\frac{\left(\lambda_{2}-\lambda_{1}\right) \beta_{1} S_{h}^{*} I_{h}^{*}+\left(\lambda_{2}-\lambda_{3}\right) I_{h}^{*}}{W_{2}}, 1\right\}, 0\right\}\right)^{2}+W_{3} \\
& \left.\times\left(\max \left\{\min \left\{\frac{\left(\lambda_{4} \epsilon_{1} S_{v}^{*}+\lambda_{5} \epsilon_{2} I_{v}^{*}\right)}{W_{3}}, 1\right\}, 0\right\}\right)^{2}\right) \\
+ & \lambda_{1} \frac{d S_{h}^{*}}{d t}+\lambda_{2} \frac{d I_{h}^{*}}{d t}+\lambda_{3} \frac{d R_{h}^{*}}{d t}+\lambda_{4} \frac{d S_{v}^{*}}{d t}+\lambda_{5} \frac{d I_{v}^{*}}{d t} .
\end{aligned}
$$

\section{Numerical Results and Discussion}

In this section, we present the numerical simulation for the proposed model with and without control system. The proposed model (1) and (2) is solved by the Runge-Kutta order 4 scheme and then we compare the control system with and without control. The optimal strategy is obtained by solving the state system and the adjoint system with the transversality conditions. In our numerical simulations, we first start to solve the control system (2) by using the RungeKutta order 4 scheme forward method in time and then solve the state equations and the adjoint system with the backward method previous iterations and the value from the characterization of system (25). The weight constants used in the objective functional are $A_{1}=0.001, A_{2}=0.30, B_{1}=100$, $B_{2}=0.03, W_{1}=100, W_{2}=140$, and $W_{3}=120$. The values of the parameters are given in Table 2 . 
TABLE 2: Parameter values used in the numerical simulations of the optimal control.

\begin{tabular}{llcc}
\hline Notation & Parameter description & Value & Reference \\
\hline$b_{1}$ & Recruitment rate for human & 1.6 & {$[12]$} \\
$\lambda_{h}$ & Proportionality constant & 0.066 & {$\left[.6 \times 10^{-5}\right.$} \\
$\mu_{h}$ & Natural death rate of human & $1.8 \times 10^{-3}$ & {$[1]$} \\
$\gamma_{v}$ & Natural death rate of vector & $1.0 \times 10^{-5}$ & {$[12]$} \\
$\delta_{h}$ & Death rate due to disease at human class & $2.7 \times 10^{-3}$ & {$[26]$} \\
$\gamma_{h}$ & Recovery rate of the infection & $1.82 \times 10^{-3}$ & {$[26]$} \\
$\delta_{v}$ & Death rate at infected class of vector & 1.9 & Assumed \\
$b_{2}$ & Birth rate of vector & 0.0089 & Assumed \\
$\beta_{2}$ & Transmission between $S_{h}$ and $I_{v}$ & 0.0079 & Assumed \\
$\beta_{3}$ & Transmission between $S_{v}$ and $I_{h}$ & 0.00013 & Assumed \\
$\beta_{1}$ & Transmission coefficient between $S_{h}$ and $I_{h}$ & 0.0027 & {$[12]$} \\
$\gamma_{v}$ & Natural death rate of vector & &
\end{tabular}

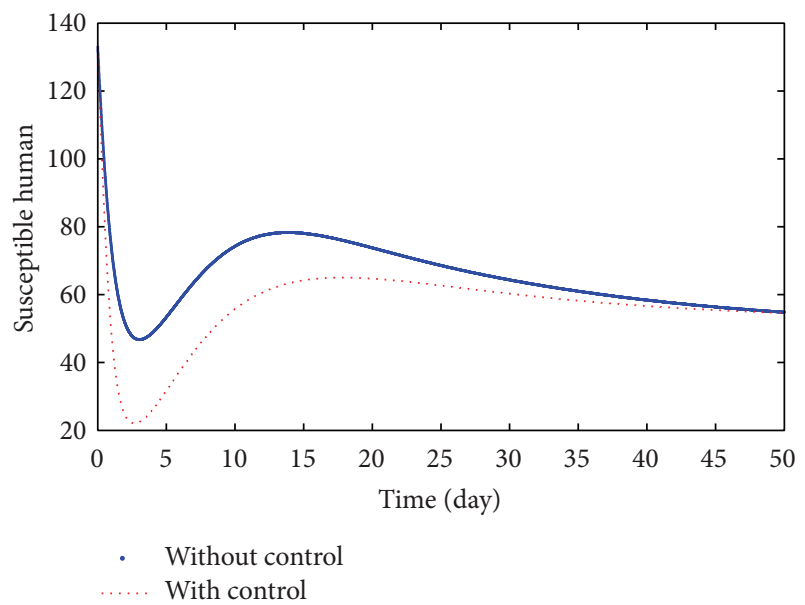

Figure 2: The plot shows the population of susceptible human with and without control.

Figures 2 and 3 represent the susceptible and infected human population. In these plots the dotted line represents the control system and the bold line shows the system of without control. In Figure 2 the dotted line shows the control in the population of susceptible human and the bold line shows the population of susceptible human with control. The dotted line decreases sharply than that of the bold line. Figure 3 represents the plot for the population of infected human for both the systems with and without control. The bold line shows the population of infected human without control and the dotted line shows the population of infected human with control. The dotted line decreases sharply as compared to the bold line; it means that the population of infected individuals decrease in the control system.

In Figure 4 the plot represents the population of recovered individuals of the two systems. The bold line shows the population of the system of without control and the dotted shows the population of the system with control. The population of recovered individuals decrease sharply as

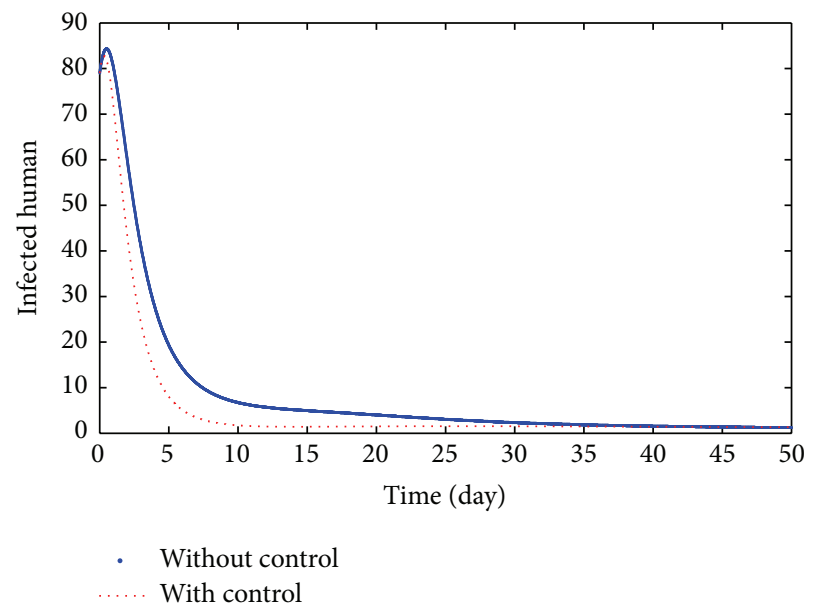

FIGURE 3: The plot shows the population of infected human with and without control.

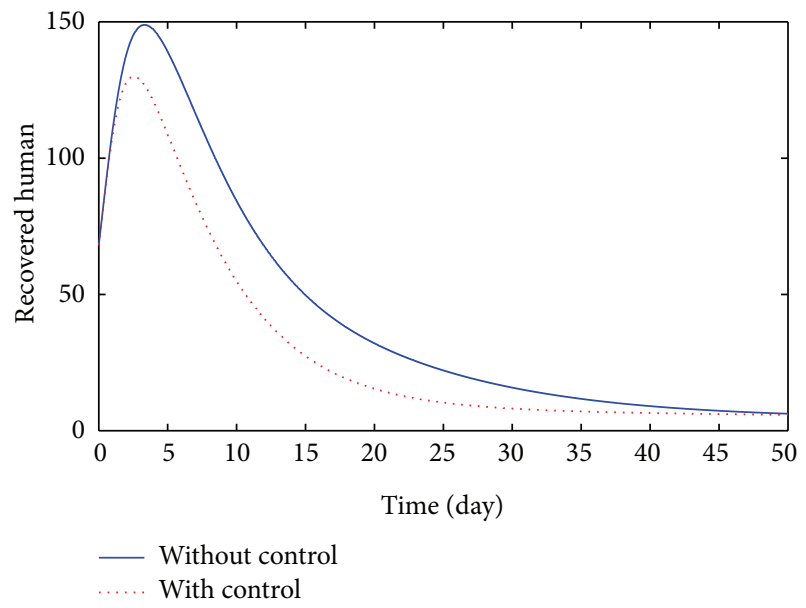

FIGURE 4: The plot shows the population of recovered human with and without control. 


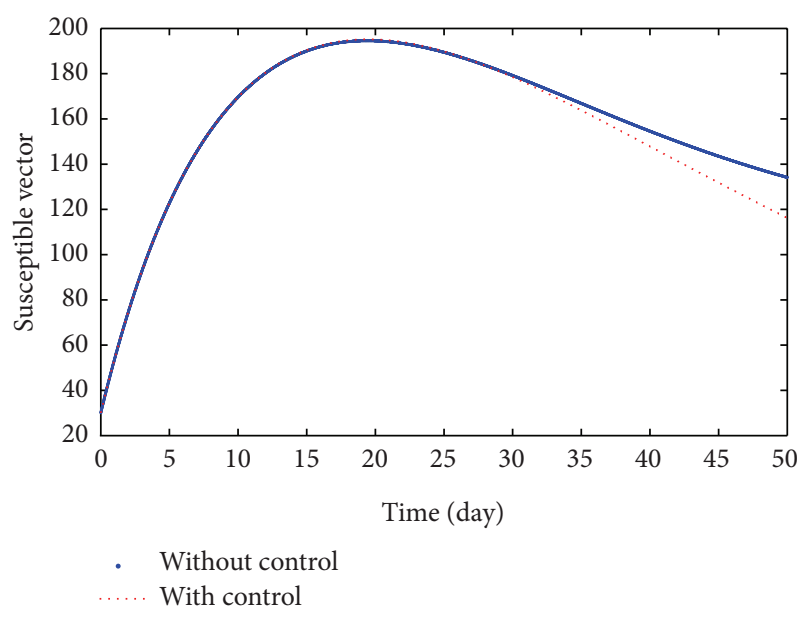

FIgURE 5: The plot shows the population of susceptible vector with and without control.

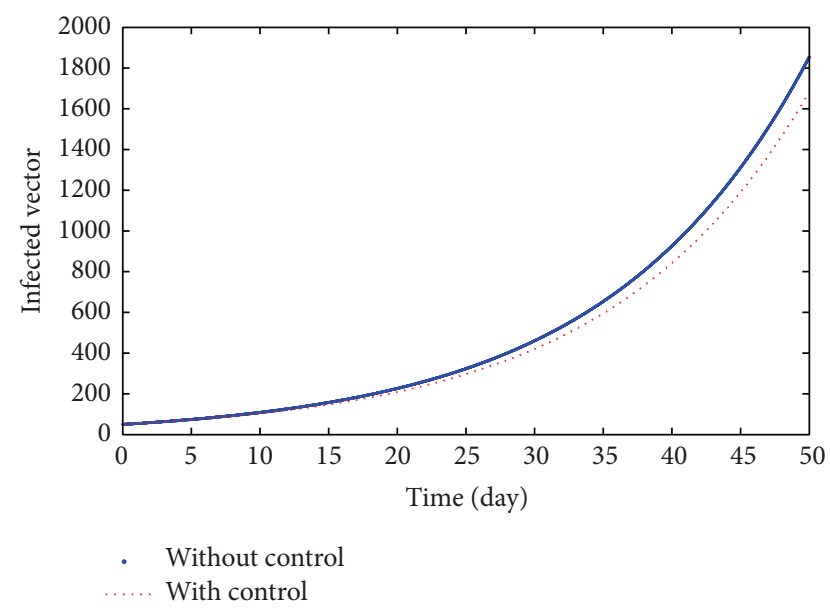

FIGURE 6: The plot shows the population of infected vector with and without control.

compared to the system of without control. Figures 5 and 6 represent the population of susceptible vector and infected vector, respectively. Figure 5 represents the population of the vector and in two systems, with and without control. The dotted line in Figures 5 and 6 shows the population of the vector in the control system. The dotted line in the Figures 5 and 6 shows the population of the vector without control system. The population of susceptible vector in the Figure 5 decreases a little bit than that of the dotted line. The population of infected vector in the Figure 6 decreases sharply than that of the dotted line.

\section{Conclusion}

In this paper, we studied the interaction of two nonlinear systems of which one is human and the other one is vector.

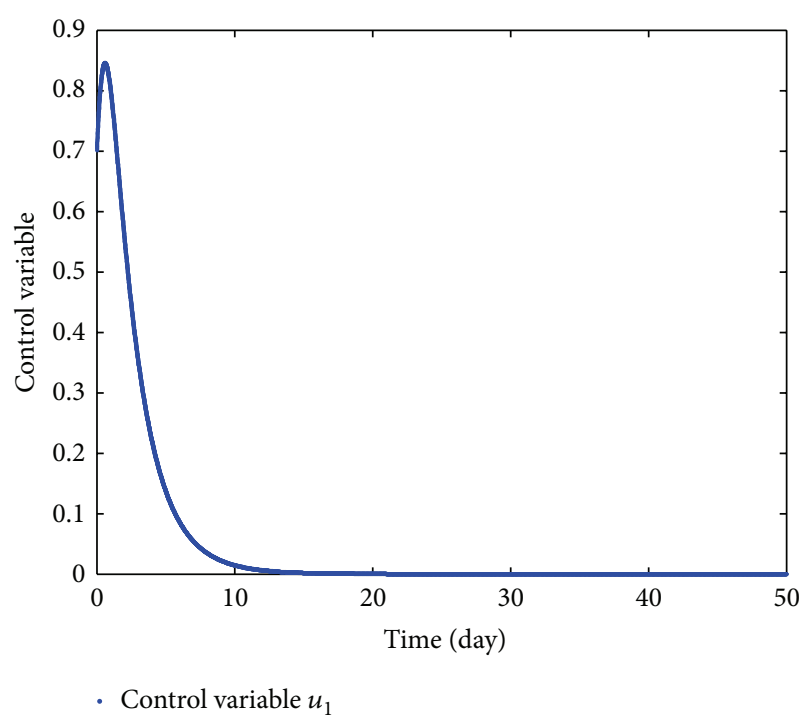

Figure 7: The plot shows the control variable $u_{1}$.

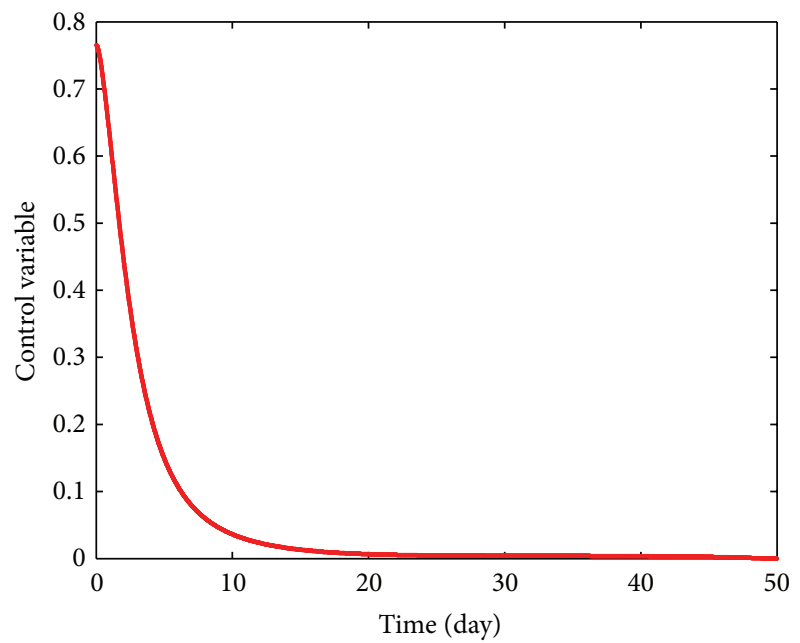

- Control variable $u_{2}$

FIGURE 8: The plot shows the control variable $u_{2}$.

The theoretical studies for the optimal control problem and their numerical simulation are presented in the paper. We used the optimal control strategies to minimize the infected human, infected vector and to maximize the population of susceptible human. The model which is developed from the numerical simulations of the optimality system showed that the population of infected human, infected vector decreases and the population of susceptible human increases. We also showed that with certain values of control rates there exist their corresponding optimal solutions.

\section{Conflict of Interests}

The authors declare that there is no conflict of interests regarding the publication of this paper. 


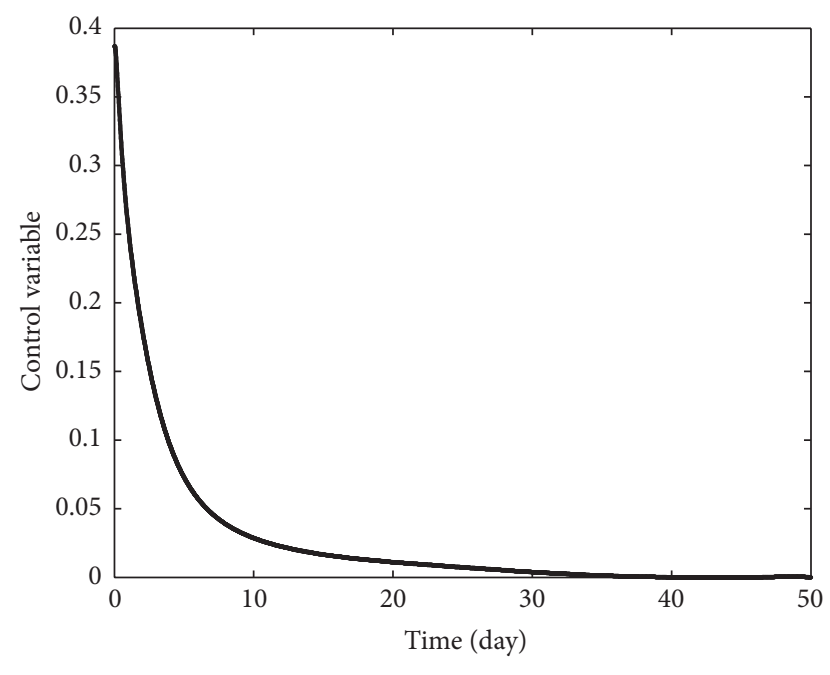

- Control variable $u_{3}$

FIGURE 9: The plot shows the control variable $u_{3}$.

\section{Acknowledgments}

The authors are thankful to the honorable editor and the reviewers for their careful reading and suggestions which greatly improved the presentation of this work.

\section{References}

[1] R. U. M. Palaniappan, S. Ramanujam, and Y. Chang, "Leptospirosis: pathogenesis, immunity, and diagnosis," Current Opinion in Infectious Diseases, vol. 20, no. 3, pp. 284-292, 2007.

[2] R. Inada and Y. Ido, "Etiology mode of infection and specific therapy of Weil's disease," The Journal of Experimental Medicine, vol. 23, pp. 377-402, 1916.

[3] R. C. Abdulkader, A. C. Seguro, P. S. Malheiro, E. A. Burdmann, and M. Marcondes, "Peculiar electrolytic and hormonal abnormalities in acute renal failure due to leptospirosis," The American Journal of Tropical Medicine and Hygiene, vol. 54, no. 1, pp. 1-6, 1996.

[4] V. M. Arean, G. Sarasin, and J. H. Green, "The pathogenesis of leptospirosis: toxin production by leptospira icterohaemorrhagiae," The American journal of veterinary research, vol. 28, pp. 836-843, 1964.

[5] V. M. Arean, "Studies on the pathogenesis of leptospirosis. II, a clinicopathologic evaluation of hepatic and renal function in experimental leptospiral infections," Laboratory Investigation, vol. 11, pp. 273-288, 1962.

[6] S. Barkay and H. Garzozi, "Leptospirosis and uveitis," Annals of Ophthalmology, vol. 16, no. 2, pp. 164-178, 1984.

[7] N. Chitnis, T. Smith, and R. Steketee, "A mathematical model for the dynamics of malaria in mosquitoes feeding on a heterogeneous host population," Journal of Biological Dynamics, vol. 2, no. 3, pp. 259-285, 2008.

[8] M. Derouich and A. Boutayeb, "Dengue fever: mathematical modelling and computer simulation," Applied Mathematics and Computation, vol. 177, no. 2, pp. 528-544, 2006.
[9] L. Esteva and C. Vargas, "A model for dengue disease with variable human population," Journal of Mathematical Biology, vol. 38, no. 3, pp. 220-240, 1999.

[10] P. Pongsuumpun, T. Miami, and R. Kongnuy, "Age structural transmission model for leptospirosis," in Proceedings of the $3 \mathrm{rd}$ International Symposium on Biomedical Engineering, pp. 411416, 2008.

[11] W. Triampo, D. Baowan, I. M. Tang, N. Nuttavut, J. WongEkkabut, and G. Doungchawee, "A simple deterministic model for the spread of leptospirosis in Thailand," International Journal of Biomedical Science, vol. 2, pp. 22-26, 2007.

[12] G. Zaman, "Dynamical behavior of leptospirosis disease and role of optimal control theory," International Journal of Mathematics and Computation, vol. 7, no. 10, pp. 80-92, 2010.

[13] G. Zaman, M. A. Khan, S. Islam, M. I. Chohan, and I. H. Jung, "Modeling dynamical interactions between leptospirosis infected vector and human population," Applied Mathematical Sciences, vol. 6, no. 26, pp. 1287-1302, 2012.

[14] A. A. Lashari, K. Hattaf, G. Zaman, and X. Li, "Backward bifurcation and optimal control of a vector borne disease," Applied Mathematics \& Information Sciences, vol. 7, no. 1, pp. 301-309, 2013.

[15] A. A. N. Rafizah, B. D. Aziah, Y. N. Azwany et al., "Risk factors of leptospirosis among febrile hospital admissions in northeastern Malaysia," Preventive Medicine, vol. 57, pp. S11-S13, 2013.

[16] K. Hattaf, A. A. Lashari, Y. Louartassi, and N. Yousfi, "A delayed sir epidemic model with general incidence rate," Electronic Journal of Qualitative Theory of Differential Equations, vol. 3, pp. $1-9,2013$.

[17] A. A. Lashari, K. Hattaf, and G. Zaman, "A delay differential equation model of a vector borne disease with direct transmission," International Journal of Ecological Economics and Statistics, vol. 27, no. 4, pp. 25-35, 2012.

[18] S. Lenhart and J. T. Workman, Optimal Control Applied to Biological Models, Mathematical and computational Biology Sries, Chapman \& Hall, London, UK, 2007.

[19] K. W. Blayneh, Y. Cao, and H. D. Kwon, "Optimal control of vector-borne diseases: treatment and prevention," Discrete and Continuous Dynamical Systems B, vol. 11, no. 3, pp. 587-611, 2009.

[20] A. A. Lashari and G. Zaman, "Optimal control of a vector borne disease with horizontal transmission," Nonlinear Analysis: Real World Applications, vol. 13, no. 1, pp. 203-212, 2012.

[21] G. Zaman, Y. H. Kang, and I. H. Jung, "Optimal treatment of an SIR epidemic model with time delay," BioSystems, vol. 98, no. 1, pp. 43-50, 2009.

[22] O. Sharomi, C. N. Podder, A. B. Gumel, E. H. Elbasha, and J. Watmough, "Role of incidence function in vaccine-induced backward bifurcation in some HIV models," Mathematical Biosciences, vol. 210, no. 2, pp. 436-463, 2007.

[23] G. Birkhoff and G. Rota, Ordinary Differential Equations, John Wiley \& Sons, New York, NY, USA, 4th edition, 1989.

[24] D. L. Lukes, Differential Equations: Classical to Controlled, vol. 162 of Mathematics in Science and Engineering, Academic Press, New York, NY, USA, 1982.

[25] M. I. Kamien and N. L. Schwartz, Dynamics Optimization: The Calculus of Variations and Optimal Control in Econimics and Management, 1991.

[26] W. Tangkanakul, H. L. Smits, S. Jatanasen, and D. A. Ashford, "Leptospirosis: an emerging health problem in Thailand," Southeast Asian Journal of Tropical Medicine and Public Health, vol. 36, no. 2, pp. 281-288, 2005. 


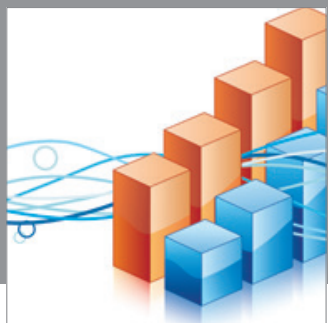

Advances in

Operations Research

mansans

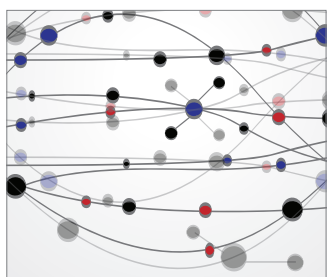

The Scientific World Journal
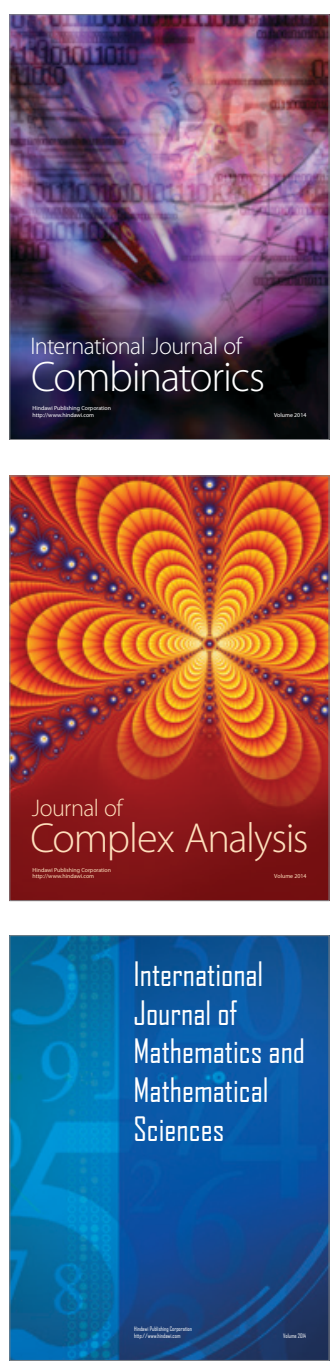
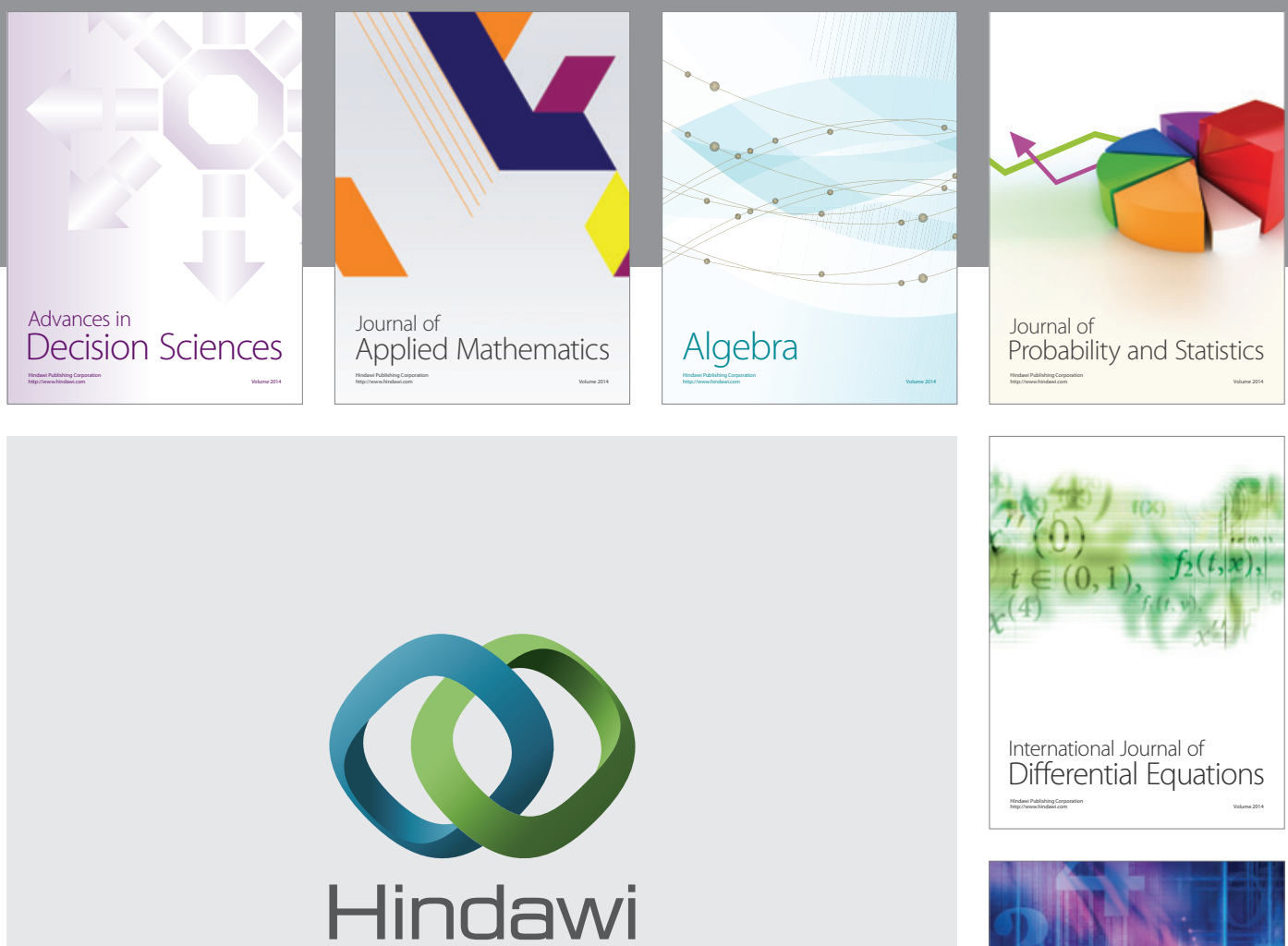

Submit your manuscripts at http://www.hindawi.com
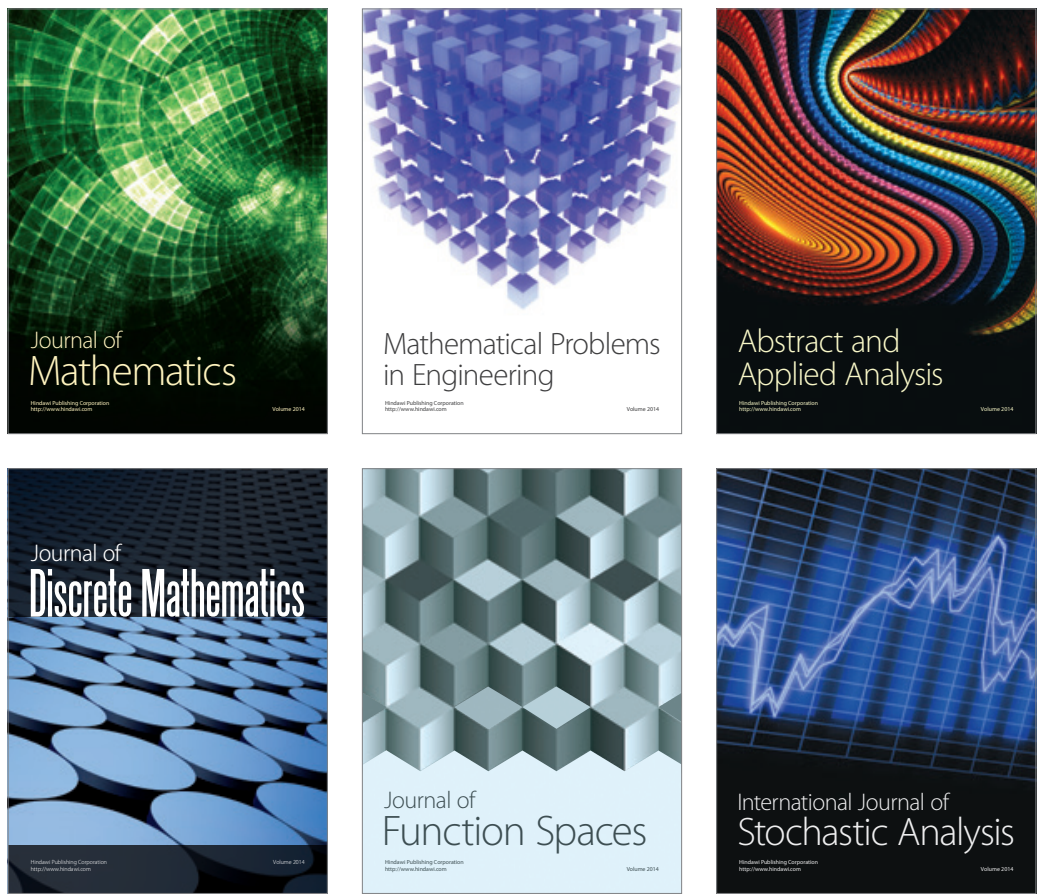

Journal of

Function Spaces

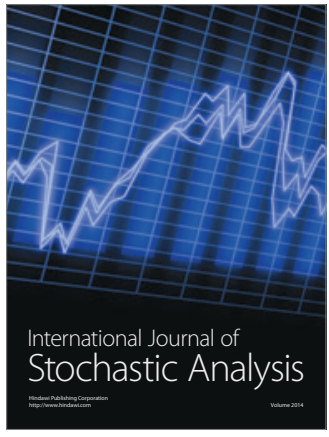

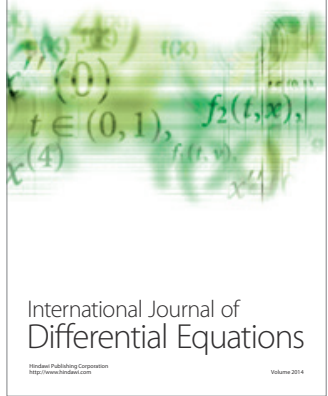
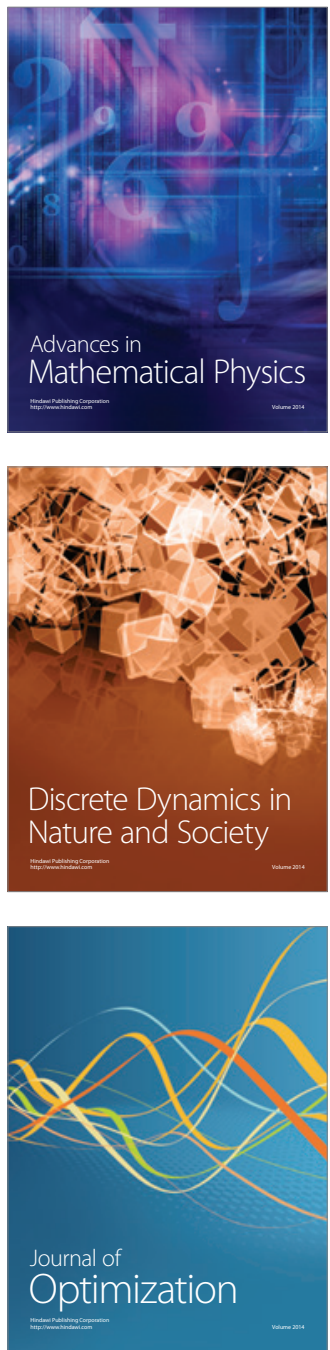